\title{
Adherence to and acceptability of artemether- lumefantrine as first-line anti-malarial treatment: evidence from a rural community in Tanzania
}

\author{
Abdunoor M Kabanywanyi ${ }^{1 *}$, Christian Lengeler², Prudensiana Kasim', Said King'eng'ena', Raymond Schlienger ${ }^{3}$, \\ Nathan Mulure ${ }^{4}$, Blaise Genton ${ }^{1,2}$
}

\begin{abstract}
Background: Controlled clinical trials have shown that a six-dose regimen of artemether-lumefantrine (AL) therapy for uncomplicated Plasmodium falciparum malaria results in cure rates $>95 \%$ with good tolerability.

Materials and methods: A prospective study was carried out to document the adherence to and acceptability of AL administration. This was undertaken in the context of the ALIVE study, a prospective, community-based, observational study in a rural, malaria-endemic area of Tanzania. Following microscopic confirmation of $P$. falciparum infection, the first AL dose was taken under supervision, with the subsequent five doses taken unsupervised at home. Patients were randomized to receive a home-based assessment close to the scheduled time for one of the unsupervised doses, but were blinded to which follow-up visit they had been allocated. A structured questionnaire was administered by trained staff and AL consumption was confirmed by inspection of blister packs.

Results: A total of 552 patients were recruited of whom 352 (63.8\%) were $<13$ years old. The randomization process allocated 112, 109, 110, 100 and 111 patients to a follow-up visit after doses 2, 3, 4, 5 and 6, respectively. For dose $2,92.0 \%$ of patients (103/112) correctly took $A L$ at $8 \pm 1$ hours after dose 1 . The remaining doses were taken within four hours of the correct time in $87-95 \%$ of cases. Nine patients (1.7\%) missed one dose. Blister packs were available for inspection in 548 of cases (99.3\%) and confirmed patient-reported data that the previous dose had been administered. Nearly all patients took AL with water (549/552 [99.5\%]). Two patients (0.4\%) took the drug with food. The dosing pictogram and clustering of tablets within the blister packs was considered helpful by $91.8 \%$ and $100.0 \%$ of patients, respectively. Overall, $87.1 \%$ of patients (481/552) found AL easier to take/administer than sulphadoxine-pyrimethamine (SP) and $87.7 \%$ (484/552) believed that AL was more effective than SP.
\end{abstract}

Discussion: Factors contributing to adherence were likely to be helpful packaging, pictorial dosing instructions and patients' conviction that AL is effective.

Conclusion: Adherence to the dosing regimen and timing of AL administration was very good.

\section{Background}

Malaria is the leading cause of outpatient and inpatient admissions in most sub-Saharan African countries, including Tanzania, and continues to exert a high burden in terms of mortality [1-3], morbidity $[1,4]$ and health expenditure $[5,6]$. The use of anti-malarial drugs for chemotherapy and chemoprophylaxis is a critical

\footnotetext{
* Correspondence: amulokozi@ihi.or.tz

'Ifakara Health Institute, P.O. Box 78373, Kiko Avenue, Old Bagamoyo Road,
} Mikocheni, Dar es Salaam, Tanzania component in the fight against malaria, but Plasmodium falciparum resistance to conventional anti-malarials, such as chloroquine and sulphadoxine-pyrimethamine (SP), is high [1,7-10]. It is for this reason that the World Health Organization (WHO) has been recommending artemisinin-based combination therapy (ACT) as firstline therapy to replace failing anti-malarial drugs [11].

The ACT artemether-lumefantrine (AL, Coartem $\left.{ }^{\circ}\right)$ combines the short-acting artemisinin derivative artemether with long-acting lumefantrine. Giving the second

\section{C) Biomed Central}


dose of AL eight hours after the first dose quickly achieves and maintains the blood concentration of artemether above the minimum effective concentration [12] to help ensure that malaria parasites are exposed to high levels during the middle third of their life cycle, when they are most susceptible to anti-malarial agents [13]. AL has demonstrated a high level of efficacy and a good tolerability profile [14-18]. As a result, the national treatment policy of Tanzania was revised in November 2006 to adopt AL as first-line treatment for uncomplicated malaria.

$\mathrm{AL}$ is administered as a six-dose regimen over a period of three days [19]. Controlled trials using this regimen have demonstrated cure rates of over 95\% [20-23], consistent with recommendations from WHO that cure rates for uncomplicated $P$. falciparum malaria should be at least $90 \%$ and preferably exceed $95 \%$ [11]. Evidence regarding the adherence to the $\mathrm{AL}$ dosing regimen and feasibility of its use in programmatic conditions remains limited [24-29]. Nevertheless, proper evaluation of adherence and acceptance outside the context of controlled clinical studies is important as AL is widely deployed throughout malaria endemic sub-Saharan countries.

Following the inclusion of AL within the national Tanzanian treatment policy, the ALIVE ('artemetherlumefantrine in vulnerable patients: exploring health impact') study was undertaken in a rural, malariaendemic area of the country with the aim of evaluating the impact of introducing AL as first-line malaria treatment on malaria-related morbidity and mortality. As part of ALIVE, a prospective study was conducted under routine conditions to document the adherence to and acceptability of AL drug administration in this setting.

\section{Methods}

\section{The ALIVE study}

ALIVE is a prospective, observational, community-based, longitudinal, demographic surveillance study taking place in two rural districts of Tanzania (Ulanga and Kilombero). The primary objective is to assess the effect of AL on all-cause mortality in infants and children $\geq 3$ months of age (and $>5 \mathrm{~kg}$ ) and $<5$ years old, using historical data based on the former first-line treatment with SP as comparator. Secondary objectives include assessment of adherence to the AL regimen, knowledge of correct AL intake and patient satisfaction.

The conduct of the ALIVE study complies with the Declaration of Helsinki. The study protocol was approved by the institutional review board of the Ifakara Health Institute (IHI), which implements the ALIVE study, and the Tanzanian National Institute for Medical Research (NIMR).

\section{Administration of AL}

As per the instructions from the manufacturer (Novartis Pharma AG, Basel, Switzerland), AL is dosed according to body weight: 5 to $<15 \mathrm{~kg}$, one tablet per dose; 15 to $<25 \mathrm{~kg}$, two tablets per dose; 25 to $<35 \mathrm{~kg}$, three tablets per dose; $\geq 35 \mathrm{~kg}$, four tablets per dose. The first two doses of AL are to be given eight hours apart on day 1 . On days 2 and 3, AL is to be given twice daily, 12 hours apart; with the morning dose being administered 24 hours after the first dose was taken.

In this study, the first dose of AL was taken under supervision at the health facility, with the subsequent five doses taken unsupervised at home. Dosing and time of taking/administering the drugs was explained and the time was clearly marked on the AL blister packs by the dispensing healthcare provider. Patients or caregivers were advised to ensure AL was not taken/given on an empty stomach.

\section{Assessment of adherence and acceptability}

Assessment of adherence to and acceptability of AL was conducted within the context of the ALIVE study and was undertaken at the Mlimba Health Center in the Kilombero District during the period March to April 2008 , i.e. approximately one year after the new treatment policy was introduced in Tanzania (January 2007). Patients living in villages no further than six kilometers from the Mlimba Health Center were eligible to take part in the assessment if they had no clinical signs of complicated malaria and P. falciparum infection was confirmed by blood smear. Informed consent was obtained from all patients.

A computer-generated randomization list was used to allocate eligible patients to a home visit for one of the five doses of AL to be taken after the initial, supervised dose. It was planned that all patients be visited at home at a time close to the scheduled time of AL administration. In the event that patients were due to be visited late in the evening or at night following an evening dose, visits were scheduled for the following morning. Patients or caregivers were informed that there would be a follow-up visit but were blinded as to which of the five possible follow-up visits they had been allocated.

Each visit was undertaken by one of two IHI field workers or three local field assistants, after appropriate training based on standard AL training materials provided by the drug manufacturer (Novartis) which had been field tested by researchers from IHI in Ifakara. For patients younger than 13 years, the patient's caregiver was interviewed instead of the patient.

\section{Questionnaire}

A structured questionnaire (see Additional file 1) was developed by researchers from IHI and the Swiss 
Tropical Institute and Novartis, after which it was field tested by researchers from IHI in Ifakara, and then administered to the patient or caregiver at each visit. This included questions on the number of doses to be administered, the number of tablets per dose, the exact time at which the last dose was given, reason for any missed doses, appropriate action if a dose was vomited, consumption of concomitant food or drink at the time of AL dosing, and how patients/caregivers remembered that AL doses should be taken. Additional questions included whether the instructions/drawing on the $\mathrm{AL}$ pack were considered useful, how patients/caregivers perceived the clustered doses in the blister packs, how easy it was for them to take/administer AL, how effective they judged AL as compared to SP (the previous first-line therapy for uncomplicated malaria in Tanzania), and their preference for AL over other treatments (antibiotics, analgesics/antipyretics, quinine injection, herbs from traditional healer or remedy from witchdoctor). Consumption of the dose was confirmed by inspection of the AL blister packs.

\section{Results}

\section{Patient population}

A total of 552 patients met the eligibility criteria and were recruited for the study (Table 1$)$. The majority of patients $(352 / 552[63.8 \%])$ were aged less than 13 years. All included patients had come to the medical facility to seek medical attention for fever. Almost all patients (544/552, 98.6\%) were reported to be unwell or moderately unwell at the time of presentation to the health facility.

The randomization process allocated 112, 109, 110, 100 and 111 patients to a follow-up visit after doses 2, $3,4,5$ and 6 , respectively.

\section{Adherence to AL}

AL was dispensed at the Mlimba Health Center in all cases. When asked, $100 \%$ of patients/caregivers reported that they had received an explanation of how to use AL. Results of the questionnaire confirmed that all patients/ caregivers understood the number of doses required, and the number of tablets that should be taken/administered per dose (Table 2). All but one patient responded that five doses in total were to be taken, an answer that was correct when referring only to the doses for which they were responsible (the first dose was given by the healthcare provider). AL was taken at the correct time in approximately $90 \%$ of cases for each dose (Table 2). For dose 2, 92.0\% of patients (103/112) took AL at $8 \pm 1$ hours after dose 1 . The remaining doses were taken within four hours of the correct time in $87-95 \%$ of cases. In total, nine out of 522 patients (1.7\%) reported missing a single dose of AL. In two out
Table 1 Patient characteristics $(n=522)$

\begin{tabular}{ll}
\hline Attribute & N (\%) \\
\hline Age & \\
$<13$ years & $352(63.8)$ \\
$\geq 13$ years & $200(36.2)$ \\
\hline Female gender & $319(57.8)$ \\
\hline Level of education of patient/caregiver & \\
None & $63(11.4)$ \\
Primary school & $421(76.3)$ \\
Secondary school & $68(12.3)$ \\
College & $0(0)$ \\
\hline Occupation of patient/caregiver & \\
Employed & $22(4.0)$ \\
Self employed & $77(13.9)$ \\
Farmer & $369(66.9)$ \\
Other & $84(15.2)$ \\
\hline Age & \\
3 months - 3 years & $270(48.9)$ \\
$3-8$ years & $62(11.2)$ \\
8 - 12 years & $39(7.1)$ \\
$>12$ years & $181(32.8)$ \\
\hline Patient condition on presentation at health facility & \\
Very unwell & $0(0)$ \\
Unwell & $399(72.2)$ \\
Moderately unwell & $145(26.3)$ \\
Moderately well & $2(0.4)$ \\
Well & $1(0.2)$ \\
Perfectly well & $5(0.9)$ \\
\hline
\end{tabular}

of these nine cases, the patient had forgotten to take the final dose. One patient used the intended dose to replace a dose vomited previously, and one patient ceased to take the drug after vomiting. In the remaining five patients there was no apparent reason for missing prescribed doses. AL blister packs were available for examination at the randomized visit in 548 cases (99.3\%), and the reported number of doses taken corresponded with actual pill count at each visit. No patient missed more than one dose, and no patient missed dose 2. In case of a dose being vomited, the majority of patients correctly understood that they should return to the health facility for a replacement dose $(316 / 552$, $57.3 \%)$. However, a relatively high proportion of patients (42.7\%) incorrectly believed that a replacement dose could be taken from the existing blister pack or that no action was required.

Nearly all patients took AL with water (549/552 [99.5\%]). Two patients (0.4\%) took the drug with food. The most frequently reported factor that positively influenced adherence to the timing of AL dosing was the impact of the current illness (Table 2). 
Table 2 Assessment of adherence to the AL dosing regimen, as evaluated by questionnaire $(n=522)$

\begin{tabular}{ll}
\hline \multicolumn{2}{l}{ How many doses in total to be administered for a } \\
complete course of treatment \\
1 \\
2 \\
3 & 0 \\
4 & 0 \\
5 & 0 \\
6 & 0 \\
\hline Number of tablets per dose to be taken? & $551(99.8)$ \\
1 tablet & $1(0.2)$ \\
2 tablets & $270 / 270$ \\
3 tablets & $(100)$ \\
4 tablets & $62 / 62(100)$ \\
& $40 / 40(100)$ \\
\hline
\end{tabular}

AL dose taken at correct time (i.e. \pm 1 hours for dose $2, \pm$ 4 hours for doses 3-6)

\begin{tabular}{ll} 
Dose 2 & $103 / 112$ \\
& $(92.0)$ \\
Dose 3 & $103 / 109$ \\
& $(94.5)$ \\
& $100 / 110$ \\
Dose 4 & $(91.0)$ \\
& $96 / 110$ \\
Dose 5 & $(87.0)$ \\
& $99 / 111$ \\
Dose 6 & $(89.2)$ \\
\hline Number of missed doses & \\
Dose 2 & $0 / 122(0)$ \\
Dose $3^{\mathrm{a}}$ & $2 / 109(1.8)$ \\
Dose $4^{\mathrm{b}}$ & $2 / 110(1.8)$ \\
Dose $5^{\mathrm{c}}$ & $3 / 100(3.0)$ \\
Dose $6^{\mathrm{d}}$ & $2 / 111(1.8)$ \\
\hline Action to be taken $f$ tablets are vomited
\end{tabular}

Action to be taken if tablets are vomited

Go back to health facility for replacement dose $\quad 316$ (57.3)

Give another dose 209 (37.8)

Do nothing $27(4.9)$

Don't know $0(0)$

\begin{tabular}{ll}
\hline With what was AL taken/given? & $0(0)$ \\
Nothing & $549(99.4)$ \\
Water only & $2(0.4)$ \\
Food & $1(0.2)$ \\
Beverage & $0(0)$ \\
Other & $171(31.0)$ \\
\hline Timing of tablet intake when administered with food? & $2(0.4)$ \\
$\quad$ Before meal & $379(68.6)$ \\
During meal & \\
After meal & $231(41.8)$ \\
\hline What acted as a reminder to take tablets? & $309(55.9)$ \\
The dispenser's instructions &
\end{tabular}

Table 2: Assessment of adherence to the AL dosing regimen, as evaluated by questionnaire $(n=522)$ (Continued)

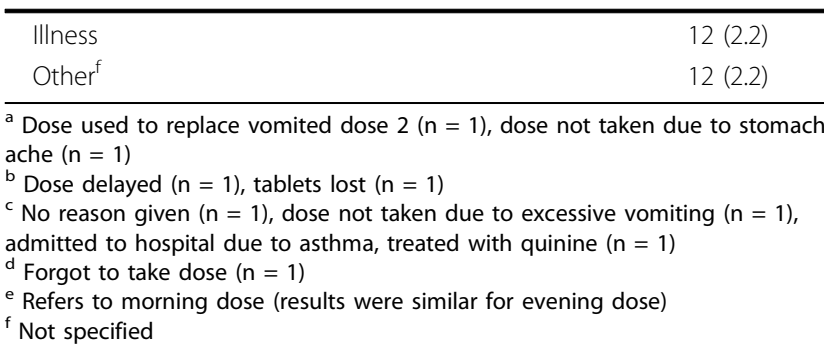

\section{Acceptability of AL}

Almost all patients (91.8\%) found the dosing pictogram helpful, and all patients reported that clustering of tablets within the blister packs was useful (Table 3). In total, $87.1 \%$ of patients $(481 / 552)$ found AL easier to take/administer than SP and 484/552 (87.7\%) believed that AL was more effective than SP (Table 3). Approximately $90 \%$ of patients $(495 / 552)$ would not have preferred other medications than AL to treat the current illness, although 5.6\% (31 patients) would have chosen quinine injections. Two patients would have preferred to receive herbs from a traditional healer.

\section{Discussion}

Adherence to the standard AL regimen was very good in this population of patients in a rural area of sub-Saharan Africa. The full six-dose regimen was taken by $98 \%$ of patients, with the dose being taken at a satisfactory time in $\sim 90 \%$ of cases. These results were obtained without any additional training for staff at the dispensing health center beyond the standard National Malaria Control Programme training initiative, and no special guidance was given to patients other than that which is routinely offered by the local healthcare personnel. The proportion of patients taking all five unsupervised doses was consistent with other reports for ACT in general [24,25] and AL in particular [26-29]. This is, however, the first study to provide data related to the timing of AL administration, and the first to apply a randomized study design to the assessment of AL adherence.

Although multiple doses of $\mathrm{AL}$ are required, there is no need for individual dose calculations according to body weight; instead, complete treatment packages are available for each body weight group. Thus, AL is simpler to prescribe than SP, the previous first-line therapy, which requires weight-adjusted dosing. However, patients need to take five doses of AL unsupervised over a three-day period compared to a single dose of SP. The findings, however, indicate that adherence to the $\mathrm{AL}$ regimen is very good following standard instructions from the dispensing healthcare provider, as confirmed 
by pill counts at the randomized visits. According to the patient responses to the questionnaire, the clustering of tablets for each dose within the AL blister packets is likely to have contributed to the correct number of tablets being taken, and the pictogram shown on the packaging was considered as a helpful supplement to the instructions provided by healthcare workers. The pictogram made the timing of drug administration clear in this rural area where few individuals have a clock or wrist watch [30]. Care should be taken, however, that dispensing staff explain that the 'sun' and 'moon' symbols refer to daytime and night-time, and not sunrise and sunset as believed in a few isolated cases among the

Table 3 Acceptability assessments $(n=552)$

\begin{tabular}{|c|c|}
\hline & $\mathrm{N}(\%)$ \\
\hline \multicolumn{2}{|c|}{ Were the instructions (drawings) in the AL pack useful? } \\
\hline Yes & $507(91.8)$ \\
\hline No & $34(6.2)$ \\
\hline Don't know & $11(2.0)$ \\
\hline \multicolumn{2}{|c|}{$\begin{array}{l}\text { Was the clustering of AL doses useful to remember how to } \\
\text { take the drug? }\end{array}$} \\
\hline Helpful & $552(100)$ \\
\hline Confusing & $0(0)$ \\
\hline Not important & $0(0)$ \\
\hline \multicolumn{2}{|l|}{ How do you or your child feel now? } \\
\hline Very unwell & $6(1.1)$ \\
\hline Unwell & $5(0.9)$ \\
\hline Moderately unwell & $3(0.6)$ \\
\hline Moderately well & $47(8.5)$ \\
\hline Well & $486(88.0)$ \\
\hline Perfectly well & $9(0.9)$ \\
\hline \multicolumn{2}{|c|}{ How do you find AL to administer/take? } \\
\hline Easier to take than SP & $481(87.1)$ \\
\hline Less easy than $\mathrm{SP}$ & $2(0.4)$ \\
\hline Same as SP & $32(5.8)$ \\
\hline Don't know & $37(6.7)$ \\
\hline \multicolumn{2}{|l|}{ Do you find that AL works? } \\
\hline Yes & $549(99.5)$ \\
\hline Better than SP & $484(87.7)$ \\
\hline Same as SP & $32(5.8)$ \\
\hline Don't know & $35(6.5)$ \\
\hline No & $3(0.6)$ \\
\hline \multicolumn{2}{|c|}{$\begin{array}{l}\text { Would you or your child prefer to have anything else than } \\
\text { AL for this particular illness? }\end{array}$} \\
\hline No & $495(89.7)$ \\
\hline Yes & $57(10.3)$ \\
\hline No specific choice & $21 / 57(36.8)$ \\
\hline Antibiotics & $1 / 57(1.8)$ \\
\hline Analgesics/antipyretics & $2 / 57(3.5)$ \\
\hline Quinine injection & $\begin{array}{l}31 / 57 \\
(54.4)\end{array}$ \\
\hline Herbs from traditional healer & 2/57 (3.5) \\
\hline
\end{tabular}

study population. Finally, the wide-held response that AL is effective may also have played a role in supporting adherence to the regimen.

It was notable that responses indicated that $\mathrm{AL}$ was virtually never taken with food, despite the fact that health providers emphasized that AL works better if not taken on an empty stomach. Based on responses to the question about timing of tablet intake, it appears that some patients may have eaten shortly before or after taking the AL dose and did not consume additional food at the time of dosing, but specific information was not collected. It is encouraging, however, that a recent analysis of data from a large-scale study of AL in five African countries found that although concomitant food intake increased lumefantrine absorption in children with malaria, there was no tendency for lower food intake in the few patients in whom treatment failure was recorded [31]. Indeed, all 37 patients who were unable to eat food with any dose achieved PCR-corrected cure at day 28 . Nevertheless, food consumption, or resuming food consumption as soon as possible, at the time of AL dosing remains advisable in order to maximize effectiveness, in view of the observed association between lumefantrine exposure and clinical and parasitological outcomes [23]. This can, of course, be challenging since initially patients may be reluctant to eat due to symptoms of nausea and vomiting during the acute phase of malaria.

Fewer than $60 \%$ of patients or caregivers understood the need to return to the health clinic for a replacement if a dose was vomited. Although the number of patients in whom a dose was reported to have been vomited was low $(n=2)$, the findings that more than $40 \%$ of patients or caretakers did not know what to do if this happens is of concern.

The current findings provide detailed evidence of the timely intake of AL under programmatic conditions. Other analyses of unsupervised adherence have all involved home visits after the three-day course was completed [26-29]. Encouragingly, however, no study has reported fewer than $90 \%$ of patients taking all six doses by the end of the three-day treatment period, based on pill counts. In this case, it was possible to validate oral information about pill administration in over 99\% of cases, and found no difference between reported consumption and the remaining number of pills. Such validation is essential given the known limitations of self-reporting [32]. The findings of this study concur with those described recently by Bell et al, based on a study in Malawi in which children or adults with uncomplicated $P$. falciparum malaria were randomized to receive AL or chorproguanil-dapsone [29]. Of the patients randomized to receive $\mathrm{AL}, 100 \%$ reported correct pill consumption during oral interviews. However, 
in a subpopulation of 87 patients in whom pills were dispensed from an electronic pill container that recorded the time of opening, Bell et al found that the rate of adherence was only $92 \%$. Thus, the adherence rate obtained using this different measurement approach was the same as that observed in this study population (92\%), where we assessed adherence by randomized, scheduled visits shortly after each dose was due to be taken. Clinically, the high adherence rates observed when AL is self-administered after the first dose have been shown to result in excellent efficacy rates irrespective of whether the drug is given supervised or unsupervised [23].

Certain aspects of the study design merit consideration. The study was undertaken in the context of routine use of AL therapy in a rural area of sub-Saharan Africa. The healthcare providers who dispensed AL did not receive any special training in addition to the standard training provided through the National Malaria Control Programme, when first-line treatment with AL was introduced, approximately one year prior to this assessment. Also, the randomization approach helped ensure comparability of groups for each AL dose assessed, and minimized the influence of interviews by avoiding expected or repeated visits. However, we are aware that responses may be due to the phenomenon that patients or caregivers provided answers they thought would be expected or desired by the interviewer. Whether or not a pill had been removed from the blister pack could be checked through pill counts, but other questions - e.g. those in which the acceptability of AL was compared to other treatment options could not be validated. Furthermore, responses for children under 13 years were given by parents or caregivers, not by the patient. Therefore, the views expressed in cases of children under 13 are those of their parents/ guardians and may not necessarily reflect the patient's perspective. We also recognize that the questions posed could be refined, particularly those which resulted in $100 \%$ or near- $100 \%$ responses, such as 'Was the clustering of $\mathrm{AL}$ doses useful to remember how to take the drug?'. Moreover, there is a need to standardize questionnaires that assess adherence and acceptability to improve the quality and subtlety of the information gained and to improve comparability between studies.

In conclusion, adherence to the AL regimen as standard first-line treatment of uncomplicated $P$. falciparum malaria was high among this rural study population. Patients adhered closely to the dosing regimen, partly due to effective packaging and pictorial dosing instructions and to patients' conviction that AL is effective. These results may be helpful for future training of healthcare providers by National Malaria Control Programmes in sub-Saharan Africa before and during implementation of ACT therapy as first-line anti-malarial treatment.

\section{Conflicts of interests}

Funding for this study was provided from Novartis Pharma and Novartis Foundation for Sustainable Development. A M Kabanywanyi, B Genton and C Lengeler received honoraria and travel expense reimbursement from Novartis Pharma to present study findings at various international conferences. R Schlienger and $\mathrm{N}$ Mulure are employees of Novartis Pharma. P Kasim and $S$ King'eng'ena have no conflicts of interest. A medical writer, funded by Novartis Pharma, provided editorial support based on the draft manuscript prepared by A M Kabanywanyi.

Additional file 1: Questionnaire. COA566A2422/ALIVE: A community-
based study to assess the impact of Coartem (ALu) when used as
national policy first-line treatment on malaria mortality and morbidity in
Tanzania
Click here for file
[http://www.biomedcentral.com/content/supplementary/1475-2875-9-48-
S1.DOC]

\section{Acknowledgements}

We are grateful to the healthcare providers at Mlimba Health Center for their support. We thank F Mpolo, S Salehe and B Kasongolo for their invaluable time in conducting interviews. We extend our gratitude to all members of the Mlimba community for their participation in the study. We also thank Anne-Claire Marrast, Marc Cousin, Chemtai Kipkeu, Fiyinfolu Oladirani and Jacqueline Mills of Novartis for their contributions, as well as Alex Schulze of the Novartis Foundation for Sustainable Development for his input to the manuscript.

\section{Author details}

${ }^{1}$ Ifakara Health Institute, P.O. Box 78373, Kiko Avenue, Old Bagamoyo Road, Mikocheni, Dar es Salaam, Tanzania. ${ }^{2}$ Swiss Tropical Institute, Socinstrasse 57, Basel, CH-4056, Switzerland. ${ }^{3}$ Novartis Pharma AG, Lichtstrasse 35, Basel, CH4056, Switzerland. ${ }^{4}$ Novartis Pharma (EACA), Hospital Road, Upper Hill, Nairobi, P.O. Box 46057 - 00100 GPO, Kenya.

\section{Authors' contributions}

AMK contributed to study design, was a study investigator and drafted the manuscript for input by the other authors. CL participated in questionnaire design, data interpretation and writing of the manuscript. PK and SK undertook data collection. RS contributed to study and questionnaire design and provided input to the manuscript. NM contributed to questionnaire design and acted as the medical advisor to the project. BG contributed to study and questionnaire design and provided input to the manuscript. All authors read and approved the final manuscript.

Received: 16 October 2009

Accepted: 11 February 2010 Published: 11 February 2010

\section{References}

1. World Health Organization: World Malaria Report WHO Press, World Health Organization, Geneva, Switzerland 2008, ISBN 9789241563697.

2. Rowe AK, Rowe SY, Snow RW, Korenromp EL, Schellenberg JR, Stein C, Nahlen BL, Bryce J, Black RE, Steketee RW: The burden of malaria mortality among African children in the year 2000. Int J Epidemiol 2006, 35:691-704.

3. Breman JG, Alilio MS, Mills A: Conquering the intolerable burden of malaria: what's new, what's needed: a summary. Am J Trop Med Hyg 71(Suppl 2):1S-15S. 
4. Roca-Feltrer A, Carneiro I, Armstrong Schellenberg JR: Estimates of the burden of malaria morbidity in African in children under the age of 5 years. Trop Med Int Health 2008, 13:771-783.

5. Sachs J, Malaney P: The economic and social burden of malaria. Nature 2002, 415:680-685

6. Ayieko P, Akumu AO, Griffiths UK, English M: The economic burden of inpatient paediatric care in Kenya: household and provider costs for treatment of pneumonia, malaria and meningitis. Cost Effec Resour Alloc 2009, 7:3.

7. Djaman J, Abouanou S, Basco L, Koné M: [Limits of the efficacy of chloroquine and sulfadoxine-pyrimethamine in Northern Abidjan (Cote d'Ivoire): Combined in vivo and in vitro studies](in French). Sante 2004, 14:205-209.

8. Mugittu K, Genton B, Mshinda H, Beck HP: Molecular monitoring of Plasmodium falciparum resistance to artemisinin in Tanzania. Malar $\mathrm{J}$ 2006, 5:126.

9. Mugittu K, Abdulla S, Falk N, Masanja H, Felger I, Mshinda H, Beck HP, Genton B: Efficacy of sulfadoxine-pyrimethamine in Tanzania after two years as first-line drug for uncomplicated malaria: assessment protocol and implication for treatment policy strategies. Malar J 2005, 4:55.

10. Mugittu K, Ndejembi M, Malisa A, Lemnge M, Premji Z, Mwita A, Nkya W, Kataraihya J, Abdulla S, Beck HP, Mshinda H: Therapeutic efficacy of sulfadoxine-pyrimethamine and prevalence of resistance markers in Tanzania prior to revision of malaria treatment policy: Plasmodium falciparum dihydrofolate reductase and dihydropteroate synthase mutations in monitoring in vivo resistance. Am J Trop Med Hyg 2004, 71:696-702.

11. World Health Organization: Guidelines for the treatment of malaria. 2006http://www.who.int/malaria/docs/TreatmentGuidelines2006.pdf, Accessed 18 August, 2009

12. Kokwaro G, Mwai L, Nzila A: Artemether/lumefantrine in the treatment of uncomplicated falciparum malaria. Expert Opin Pharmacother 2007, 8:75-94.

13. White NJ: Antimalarial pharmacokinetics and treatment regimens. $\mathrm{Br} J$ Clin Pharmacol 1992, 34:1-10.

14. Falade C, Makanga M, Premji Z, Ortmann CE, Stockmeyer M, de Palacios PI: Efficacy and safety of artemether-lumefantrine (Coartem ${ }^{\oplus}$ ) tablets (sixdose regimen) in African infants and children with acute, uncomplicated malaria. Trans R Soc Trop Med Hyg 2005, 99:459-467.

15. Faye B, Ndiaye $J$, Ndiaye D, Dieng Y, Faye O, Gaye O: Efficacy and tolerability of four antimalarial combinations in the treatment of uncomplicated Plasmodium falciparum malaria in Senegal. Malar J 2007, 6:80.

16. Fanello Cl, Karema C, van Doren W, Van Overmeir C, Ngamije D, D'Alessandro U: A randomised trial to assess the safety and efficacy of artemether-lumefantrine (Coartem) for the treatment of uncomplicated Plasmodium falciparum malaria in Rwanda. Trans $R$ Soc Trop Med Hy 2007, 101:344-350

17. Omari AA, Gamble C, Garner P: Artemether-lumefantrine (six-dose regimen) for treating uncomplicated falciparum malaria. Cochrane Database Syst Rev 2005, 4:CD005564.

18. Sinclair D, Zani B, Donegan S, Olliaro P, Garner P: Artemisinin-based combination therapy for treating uncomplicated malaria. Cochrane Database Syst Rev 2009, 3:CD007483.

19. Coartem ${ }^{\circledast} /$ Riamet $^{\oplus}$ (artemether/lumefantrine) Basic Prescribing Information. Novartis Pharma AG, Basel, Switzerland. Last updated September 2007 http://www.pharma.us.novartis.com/product/pi/pdf/coartem. pdf.

20. Makanga M, Premji Z, Falade C, Karbwang J, Mueller EA, Andriano K, Hunt P, De Palacios PI: Efficacy and safety of the six-dose regimen of arthemeter-lumefrantrine in pediatrics with uncomplicated plasmodium falciparum malaria: a pooled analysis of individual patient data. Am $J$ Trop Med Hyg 2006, 74:991-998.

21. van Vugt $M$, Looareesuwan S, Wilairatana $P, M c G r e a d y ~ R$, Villegas $L$, Gathmann I, Mull R, Brockman A, White NJ, Nosten F: Artemetherlumefantrine for the treatment of multidrug-resistant falciparum malaria. Trans R Soc Trop Med Hyg 2000, 94:545-548.

22. Lefévre G, Looareesuwan S, Treeprasertsuk S, Krudsood S, Silachamroon U, Gathmann I, Mull R, Bakshi R: A clinical and pharmacokinetic trial of six doses of artemether-lumefantrine for multidrug-resistant Plasmodium falciparum malaria in Thailand. Am J Trop Med Hyg 2001, 64:247-256.
23. Piola P, Fogg C, Bajunirwe F, Biraro S, Grandesso F, Ruzagira E, Babigumira J, Kigozi I, Kiguli J, Kyomuhendo J, Ferradini L, Taylor W, Checchi F,

Guthmann JP: Supervised versus unsupervised intake of six-dose artemether-lumefantrine for treatment of acute, uncomplicated Plasmodium falciparum malaria in Mbarara, Uganda: a randomized trial. Lancet 2005, 365:1467-1473.

24. Ajayi IO, Browne EN, Garshong B, Bateganya F, Yusuf B, Agyei-Baffour P, Doamekpor L, Balyeku A, Munguti K, Cousens S, Pagnoni F: Feasibility and acceptability of artemisinin-based combination therapy for the home management of malaria in four African sites. Malar J 2008, 7:6.

25. Ajayi IO, Falade CO, Olley BO, Yusuf B, Gbotosho S, lyiola T, Olaniyan O, Happi C, Munguti K, Pagnoni F: A qualitative study of the feasibility and community perception on the effectiveness of artemether-lumefantrine use in the context of home management of malaria in south-west Nigeria. BMC Health Serv Res 2008, 8:119.

26. Chinbuah AM, Gyapong JO, Pagnoni F, Wellington EK, Gyapong M: Feasibility and acceptability of the use of artemether-lumefantrine in the home management of uncomplicated malaria in children 6-59 months old in Ghana. Trop Med Int Health 2006, 11:1003-1016.

27. Rahman MM, Donorp AM, Day NP, Lindegardh N, Imwong M, Faiz MA, Bangali AM, Kamal AT, Karim J, Kaewkungwal J, Singhasivanon P: Adherence and efficacy of supervised versus non-supervised treatment with artemether/lumefantrine for the treatment of uncomplicated Plasmodium falciparum malaria in Bangladesh: a randomised controlled trial. Trans R Soc Trop Med Hyg 2008, 102:861-867.

28. Fogg C, Bajunirwe F, Piola P, Biraro S, Checchi F, Kiguli J, Namiro P, Musabe J, Kyomugisha A, Gushtmann J-P: Adherence to a six-dose regimen of artemether-lumefantrine for treatment of uncomplicated Plasmodium falciparum malaria in Uganda. Am J Trop Med 2004 71:525-530.

29. Bell DJ, Wootton D, Mukaka M, Montgomery J, Kayange N, Chimpeni P, Hughes DA, Molyneux ME, Ward SA, Winstanley PA, Lalloo DG: Measurement of adherence, drug concentrations and the effectiveness of artemether-lumefantrine, chlorproguanil-dapsone or sulphadoxinepyrimethamine in the treatment of uncomplicated malaria in Malawi. Malar J 2009, 26:204

30. Dowse R, Ehlers M: Medicine labels incorporating pictograms: do they influence understanding and adherence?. Patient Educ Couns 2005, 58:63-70.

31. Borrmann S, Sallas WM, Marrast AC, Kern SE: Exposure to lumefantrine in infants and children receiving artemether-lumefantrine for uncomplicated malaria: impact of African diet components. American Society of Tropical Medicine and Hygiene Annual Meeting, 7-11 December 2008, New Orleans, USA .

32. Garber MC, Nau DP, Erickson SR, Aikens JE, Lawrence JB: The concordance of self-report with other measures of medication adherence: a summary of the literature. Med Care 2004, 42:649-652.

doi:10.1186/1475-2875-9-48

Cite this article as: Kabanywanyi et al:: Adherence to and acceptability of artemether-lumefantrine as first-line anti-malarial treatment: evidence from a rural community in Tanzania. Malaria Journal 2010 9:48.

\section{Submit your next manuscript to BioMed Central and take full advantage of:}

- Convenient online submission

- Thorough peer review

- No space constraints or color figure charges

- Immediate publication on acceptance

- Inclusion in PubMed, CAS, Scopus and Google Scholar

- Research which is freely available for redistribution 